\title{
Platelet-activating factor podoplanin: from discovery to drug development
}

\author{
Ai Takemoto ${ }^{1} \cdot$ Kenichi Miyata $^{1} \cdot$ Naoya Fujita $^{1}$ \\ Published online: 3 July 2017 \\ (C) The Author(s) 2017. This article is an open access publication
}

\begin{abstract}
Tumor cell-induced platelet aggregation facilitates hematogenous metastasis by promoting tumor embolization, preventing immunological assaults and shear stress, and the platelet-releasing growth factors support tumor growth and invasion. Podoplanin, also known as Aggrus, is a type I transmembrane mucin-like glycoprotein and is expressed on wide range of tumor cells. Podoplanin has a role in platelet aggregation and metastasis formation through the binding to its platelet receptor, C-type lectin-like receptor 2 (CLEC-2). The podoplanin research was originally started from the cloning of highly metastatic NL-17 subclone from mouse colon 26 cancer cell line and from the establishment of 8F11 monoclonal antibody $(\mathrm{mAb})$ that could neutralize NL-17-induced platelet aggregation and hematogenous metastasis. Later on, podoplanin was identified as the antigen of $8 \mathrm{~F} 11 \mathrm{mAb}$, and its ectopic expression brought to cells the platelet-aggregating abilities and hematogenous metastasis phenotypes. From the $8 \mathrm{~F} 11 \mathrm{mAb}$ recognition epitopes, podoplanin is found to contain tandemly repeated, highly conserved motifs, designated platelet aggregation-stimulating (PLAG) domains. Series of analyses using the cells expressing the mutants and the established neutralizing anti-podoplanin mAbs uncovered that both PLAG3 and PLAG4 domains are associated with the CLEC-2 binding. The neutralizing mAbs targeting PLAG3 or PLAG4 could suppress podoplanin-induced platelet aggregation and hematogenous metastasis through inhibiting the podoplanin-CLEC-2 binding. Therefore, these domains are certainly functional in podoplanin-mediated metastasis
\end{abstract}

Naoya Fujita

naoya.fujita@jfcr.or.jp

1 Division of Experimental Chemotherapy, The Cancer Chemotherapy Center, Japanese Foundation for Cancer Research, 3-8-31, Ariake, Koto-ku, Tokyo 135-8550, Japan through its platelet-aggregating activity. This review summarizes the platelet functions in metastasis formation, the role of platelet aggregation-inducing factor podoplanin in pathological and physiological situations, and the possibility to develop podoplanin-targeting drugs in the future.

Keywords Podoplanin $\cdot$ Plateletaggregation $\cdot$ Hematogenous metastasis · CLEC-2

\section{Platelet aggregation in hematogenous metastasis}

Many reports have suggested that platelets are associated with cancer [1, 2]. In cancer patients with advanced disease, venous thromboembolism is frequently occurred. The cancerassociated venous thromboembolism is a serious leading cause of death [3]. Adding to recurrent thromboses increase the risk for cancer, cancer patients are more likely to develop metastasis after thromboembolism experiences. These recent observations more strongly suggest that there is an association between platelet activation and cancer and that activated platelets have a role in cancer progression.

Many agents inhibiting platelet activation, such as the cyclooxygenase inhibitor aspirin, phosphodiesterase inhibitors, and prostacyclin, have been shown to suppress metastasis in experimental animal models [4]. Furthermore, a calcium channel blocker, verapamil, reportedly suppresses platelet aggregation in vitro and hematogenous metastasis and spontaneous metastasis in mouse melanoma B16 and mouse colon adenocarcinoma 26 (colon 26) cells without significantly inhibiting the growth of the primary tumors [5]. These evidences suggest that platelet aggregation has a role in hematogenous metastasis. In addition to these findings in experimental models, recent robust clinical analyses have indicated that the anti-platelet agent, aspirin, reduces the frequency of metastasis and increases survival in cancer 
patients [6, 7]. However, the suppressive effects in clinical studies of other agents - such as the anti-coagulant agent lowmolecular-weight heparin - on tumor progression remain controversial. Besides, the experimental metastasis model was developed to evaluate the relationship between tumor-induced platelet aggregation and embolization and metastasis. Tsuruo et al. performed in vivo selection in which lungs excised from mice subcatenously (s.c.) transplanted with colon 26 cells were s.c. injected to other mice repeatedly to establish a colon 26 -select line (P-select 26) that potentiated the formation of lung metastasis nodules of colon 26 and established subclones from P-select 26 [8]. The characteristics of the subclones derived from P-select 26 were analyzed, and a comparison of subclones with highly metastatic and poorly metastatic potentials showed that platelet aggregation capability was positively correlated with metastasis potential. Mahalingam et al. also isolated subclones of fibrosarcoma, some of which showed high metastatic potential and platelet aggregation ability; however, other subclones showed no correlation between metastasis ability and platelet aggregation ability [9]. Thus, the capacity of a tumor to induce platelet aggregation is among the key factors for hematogenous metastasis formation, although metastasis is not controlled by this capability alone. Lung metastasis in the clones that exhibited high metastasis potentials and platelet aggregation capacity was suppressed by the induction of thrombocytopenia induced by anti-platelet antibodies or neuraminidase or by prostacyclin treatment [9]. These findings suggest that suppressing platelet activation has potential as a treatment for metastasis. However, a more efficient approach is to target the tumor-specific pathway that activates platelets, thereby avoiding the risk for bleeding in patients before and after surgery.

So far, many pathways on how platelets promote tumor metastasis are suggested. In hematogenous metastasis, more than 99.9\% of intravasated tumor cells die in circulation because they are exposed to shear stress and eliminated by natural killer (NK) cells before reaching the parenchyma of distant tissues $[10,11]$. However, some highly metastatic tumor cells can escape these fates by evoking platelet aggregation and building tumor cellplatelet aggregates. The tumor cell-platelet aggregates are easily trapped in microvasculature possibly because of their large size and adhesiveness to vessel wall of activated platelets, which is a prerequisite step for extravasation (Fig. 1). As covered by platelets, tumor cells are prevented from shear stress and immunological elimination (Fig. 1). As reported, the immune surveillance from NK cells is suppressed by some membrane proteins expressed on platelets, such as glucocorticoid-induced tumor necrosis factor receptor-related ligand [12] and MHC class I [13], or by releasing platelet-derived growth factor (PDGF) and transforming growth factor- $\beta$ (TGF- $\beta$ ) from aggregated platelets $[14,15]$. Adding to the effects on immune surveillance, many releasates from activated platelets affect properties of tumor cells and metastatic sites (Fig. 1). The aggregated platelet-derived ATP [16], CXCL5 and CXCL7 [17], and TGF- $\beta[18,19]$ support the intravascular extravasation of tumor cells, and aggregated platelet-derived lysophosphatidic acid facilitates the preparation of pre-metastatic niches to promote bone metastasis formation [20]. Not only in the circulation, tumor cells could interact and activate platelets in the primary tumor because of leaky vessels, which suggest that platelet effects come to primary tumor cells. We need to clarify the pathway to tumor progression promoted by platelets and the effect by targeting tumor-platelet pathway on many roles of platelets in pathological but in physiological condition.

\section{Podoplanin: a novel platelet aggregation-inducing factor}

To target tumor-specific platelet activation, identification of factors contributing to tumor-platelet interaction and inducing platelet activation is important. Tumor-platelet interaction depends on several factors: integrin, sialyl Lewis ${ }^{\mathrm{x}} /$ sialyl Lewis $^{\mathrm{a}}$, and so on [1]. Tissue factor expressed by tumor cells can also activate platelets.

To elucidate the factor expressed in tumor cells that induces platelet aggregations, Watanabe et al. immunized rats with the membrane fraction of a highly metastatic subclone (NL-17) derived from the P-select 26 line [8] to establish monoclonal antibody ( $\mathrm{mAb}$ )-producing hybridomas [21]. A purified $\mathrm{mAb}$ designated 8F11 showed higher reactivity toward highly metastatic NL-17 than toward weakly metastatic NL-14, and it inhibited NL-17-induced platelet aggregation. In a mouse melanoma, the mAb showed stronger reactivity to a highly metastatic $\mathrm{B} 16 \mathrm{~F} 10$ variant than to its original cell line, B16, and it inhibited B16F10-induced platelet aggregation [22]. Furthermore, 8F11 mAb inhibited NL-17-induced experimental lung metastasis [23].

An 8F11 mAb affinity-purified $44 \mathrm{kDa}$ glycoprotein (gp44) induces platelet aggregation in vitro, and its platelet aggregation activity is reduced when it is deglycosylated by sequential treatment with neuraminidase and $O$-glycanase [24]. Glycosylation hindered protein identification using mass spectroscopy. Among accumulated annotations of proteins, mouse $\mathrm{T} 1 \alpha$ antigen was expected as a candidate for $8 \mathrm{~F} 11 \mathrm{mAb}$-reactive gp 44 . The stable expression of mouse $\mathrm{T} 1 \alpha$ antigen on the surface of Chinese hamster ovary (CHO) cells was detected with $8 \mathrm{~F} 11 \mathrm{mAb}$, and the dominant platelet-inducing factor expressed in the NL-17 subclone was identified and designated Aggrus (thereafter, podoplanin) [25]. The expression of mouse podoplanin, a human podoplanin ortholog, also induced platelet aggregation capability in $\mathrm{CHO}$ cells.

After the identification of the epitope recognized by the $8 \mathrm{~F} 11 \mathrm{mAb}$ and observations of conservation among species, the functional domain critical for platelet aggregation activity was speculated and designated the platelet aggregationstimulating (PLAG) domain (see Sect. 4). The PLAG is tandemly repeated three times in a conserved manner 
Fig. 1 Platelets promote tumor progression through the tumorinduced activation and aggregation. In the circulation, tumor cells interact with platelets and produce tumor-platelet aggregates. The aggregates covered by activated platelets resist against shear stress and suppress immunological assaults by NK cells through the display MHC class I and platelet releasates. Tumor-platelet aggregates prone to adhere and form emboli in microvasculature, which could promote metastasis formation. Platelet releasates also contribute to the formation of premetastatic niche and promote tumor growth and metastasis property, EMT/invasion which could contribute to extravasation. Primary tumor possibly interacts with platelets leaked from vessels, and then, activated/aggregated platelets contribute to tumor progression through releasing factors same as in the circulation

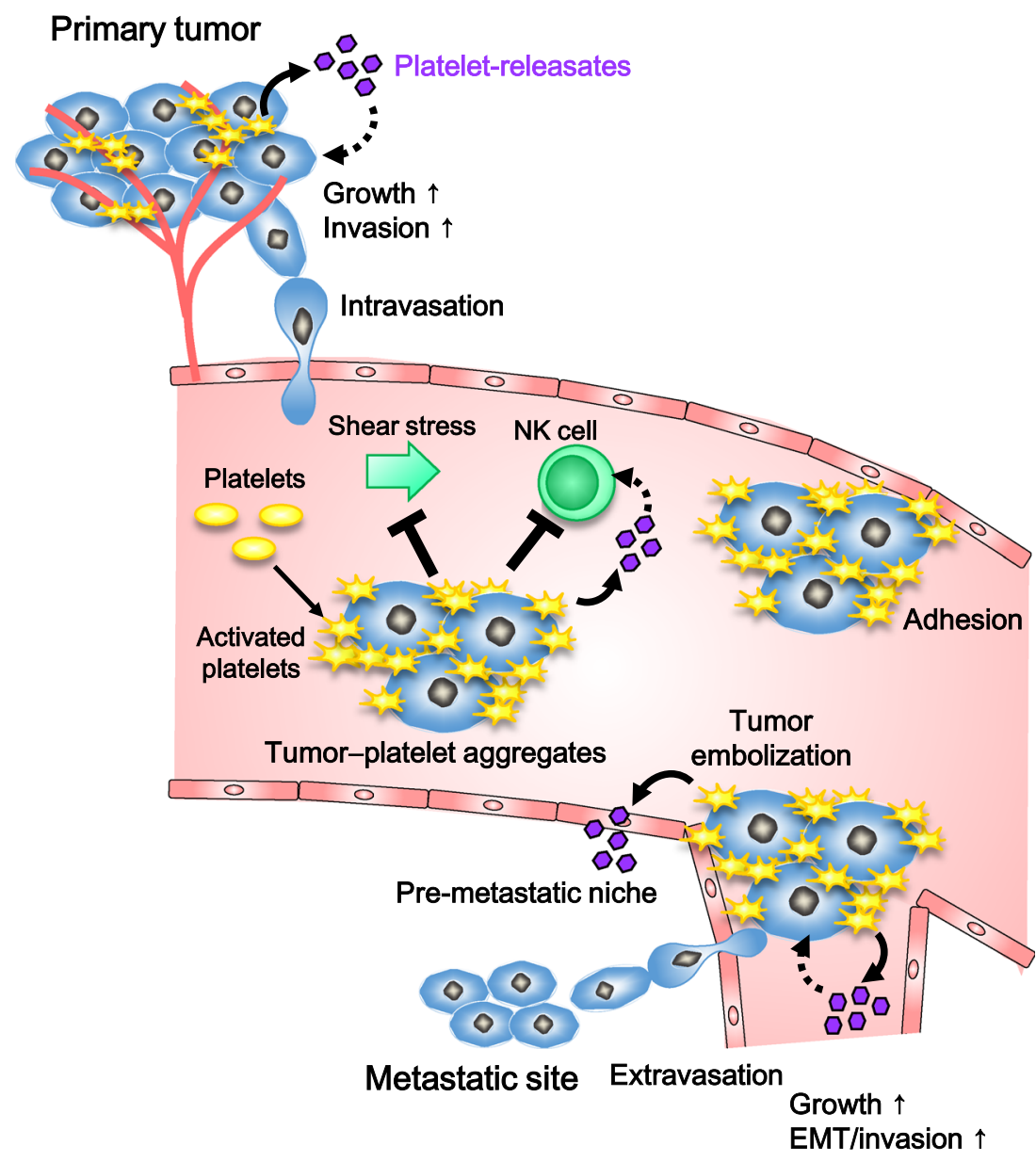

(PLAG1-3) [26]. Possible $O$-glycosylation sites at Thr34 in PLAG1 and Thr52 in PLAG3 have been suggested to be important for podoplanin-dependent platelet aggregation [25]. Podoplanin is a type I transmembrane sialomucin-like glycoprotein expressed on the cell surface of various tumors and some normal tissues (see Sect. 3). Before its identification as a factor promoting tumor metastasis through platelet aggregation, it had been discovered independently in various mammalian species and given different names: T1alpha as a water channel in humans, mice, and rats [27]; gp40 in dogs [28]; gp36 as a vascular endothelial glycoprotein in humans [29]; OTS-8 as a tumor marker [30]; 8.1.1 mAb antigen [31]; M2A as a D2-40 antigen [32]; and PA2.26 in mice [33] among others. These designations are synonymous with podoplanin.

\section{Pathological and physiological functions of podoplanin}

\subsection{Physiological expression and function}

For normal condition, podoplanin is expressed on lymphatic endothelial cells, alveolar epithelial type I cells in lung, kidney podocytes, lymph node-derived fibroblastic reticular cells (FRCs), and central nervous system [31, 34-38]. Physiologically podoplanin functions during development, as podoplanin null mice show increased embryonic lethality with disorder in heart development [39] or die after birth owing to respiratory failure and not inflated lung [40, 41]. The defect in the separation of the blood lymphatic vessels is indicated as one of the leading cause [42]. And the C-type lectin-like receptor 2 (CLEC-2) null mice also show the defect in blood lymphatic vessel separation, and the relation of lymphatic podoplaninmediated platelet aggregation is suggested [43]. In addition, the physiological functions of podoplanin are suggested in postnatal stage. For immune surveillance, lymphocytes enter lymph nodes through specialized blood vessels named high endothelial venules (HEVs). Podoplanin has a role in maintaining HEV barrier function, as the podoplanin deficiency exhibits loss of HEV integrity and spontaneous bleeding in lymph nodes. In this function, the sphingosine-1-phosphate release during podoplaninmediated platelet aggregation is indicated as a key in maintaining the integrity of HEV [44]. Not only through the platelet interaction, but CLEC-2-expressed dendritic cells cause stretching of stroma by affecting podoplanin-expressed FRC, which leads to lymph node expansion in immune response [45]. And also the 
interaction between CLEC-2 on megakaryocytes and podoplanin on FRC-like stroma cells is suggested to promote megakaryocyte expansion and proplatelet formation in bone marrow [46]. Recent accumulated reports suggest physiological functions of podoplanin in the development of lymphatic vessels, lymph nodes, and immune responses. We need to know more details about the mechanism for targeting podoplanin in cancer (see Sect. 5).

\subsection{Pathological expression and function}

Pathologically, enhanced expression of podoplanin in advanced atherosclerotic lesions is suggested to contribute to thrombus formation leading to cardiovascular events [47]. And, podoplanin expression on Th17 cells has a role in the formation of ectopic lymphoid follicles in chronic autoimmune inflammatory diseases [48]. Podoplanin is expressed on various tumor cells, including squamous cell carcinomas (SCCs), glioblastoma, osteosarcoma, bladder carcinoma, mesothelioma, and seminoma [32, 49-53], and its expression correlates with poor prognosis in brain and lung tumors [54-56]. Furthermore, podoplanin expression level correlates with the metastasis in oral SCC and bladder tumors [51, 57]. Not only in tumor cells themselves but also in cancerassociated fibroblasts (CAFs), podoplanin expression is observed and correlated with tumor malignancy and poor prognosis in lung, breast, pancreatic, and liver cancer [58-61]. Podoplanin-expressed CAFs contribute the resistance to epidermal growth factor receptor (EGFR) tyrosine kinase inhibitor gefitinib [62]. Podoplanin-expressed lymph node stromal cells enhance tumor growth in vivo by eliminating $\mathrm{CD}^{+}$ tumor-infiltrating lymphocytes that limit the efficiency of tumor immunotherapy [63]. Many reports suggest that podoplanin promotes tumor and metastasis; however, there are several controversial reports that podoplanin expression in lung SCC correlates with lower incidence of lymph node metastasis and good prognosis [64-66].

We described suggested function of activated and aggregated platelets in tumor progression and metastasis in Sect. 1. As described in Sect. 2, podoplanin is a platelet aggregationstimulating factor; thus, podoplanin expressed in tumor cells could contribute to all pathways induced by platelet aggregation (Fig. 1). In fact, the dependency on podoplanin of tumor embolization [51, 67], and the release of several platelet factors [19, 68, 69], was indicated. The PDGF release by podoplanin-mediated platelet aggregation enhances in vitro growth and the resistance for apoptosis through activation of PDGFR-PI3K/Akt pathway in osteosarcoma cells [68]. The TGF- $\beta$ release by podoplanin-dependent platelet aggregation promotes epithelial-mesenchymal transition (EMT) and invasion of urinary bladder and lung SCC cells [19]. The EGFR ligands containing EGF released by podoplanin-induced platelet aggregation promote lung SCC tumor in vivo through the EGFR signal activation [69]. These are reasonable as podoplanin acts in the initial step of those pathways induced by platelet aggregation. Moreover, some platelet-released growth factors, such as TGF- $\beta$, basic fibroblast growth factor, and EGF, promote the expression of podoplanin [70]. Thus, the release of these growth factors may further accelerate podoplanin-mediated platelet aggregation and promote tumor growth and metastasis.

\section{Functional domains and the post-translational modification of podoplanin}

\subsection{PLAG domains and glycosylation}

The PLAG1-3 domains of podoplanin are found as tandemly repeated in a conserved manner, but their contribution in inducing platelet aggregation differs over mammalian species [26]. Possible $O$-glycosylation sites, Thr34 in PLAG1 of mouse podoplanin and Thr34 in PLAG1/Thr52 in PLAG3 of human podoplanin, are implicated in platelet aggregation $[25,26]$. Sialyl $O$-glycosylation in podoplanin is central to platelet aggregation-inducing activity, as indicated by studies using enzymatic deglycosylation [24] and podoplaninexpressing $\mathrm{CHO}$ mutant series which are deficient in glycosylation pathways [71, 72].

Analyses of the glycosylation site and structure of podoplanin with lectin blot, mass spectrometry, and Edman degradation [71, 72] have revealed that podoplanin has a disialyl-core 1 structure. This sialyl $O$-glycan structure could be introduced into human podoplanin via a genetic engineered yeast strain followed by in vitro sialylation, and thereafter, the glycosylated podoplanin induced platelet aggregation [73]. A structural analysis of the complex of the sialyl $O$-glycosylated podoplanin PLAG2/3 peptide using the engineered glycosylation system and the extracellular domain of podoplanin counterpart on platelets, CLEC-2, has been reported [74]. From that, Glu47, Asp48, and sialyl-glycosylated Thr52 in the PLAG3 domain were shown to interact with CLEC-2. However, the PLAG3-mutated podoplanin still exhibited platelet aggregation-inducing ability [75]. To answer the discrepancy, another conserved region located at distant with repeated PLAG1-3 domains was identified in human podoplanin as a critical domain for podoplanin-induced platelet aggregation, and designated PLAG4. The analyses using PLAG-mutated podoplanin indicated that PLAG4 domain dominantly contributes human podoplanin-induced platelet aggregation than PLAG3, and the binding of human podoplanin to CLEC-2 depends on PLAG3 and PLAG4 domains [75]. Similarly to PLAG3, the contribution of Glu81, Asp82, and Thr85 of PLAG4 in the CLEC-2 binding was indicated. Thus, "Glu-Asp-(X)-X-X-Thr (ED[X]XXT)" could 
be the motif required for CLEC-2 binding though sialyl glycosylation in Thr85 of PLAG4 has not been shown.

\subsection{Molecular identification of CLEC-2 as a natural podoplanin receptor}

CLEC-2 was identified functionally as a platelet receptor for platelet aggregation-inducing snake venom, rhodocytin $[76,77]$. Similarities between podoplanin-induced and rhodocytin-induced platelet activation signal pathway in Src kinase and phospholipase $\mathrm{C} \gamma 2$ (PLC $\gamma 2$ ) dependency suggested that they share the same receptor. And analyses showed that podoplanin is an in vivo ligand of CLEC-2 $[78,79]$. Now, the downstream cascade of podoplanin/ CLEC-2 leading to platelet activation is indicated. When podoplanin or rhodocytin binds to CLEC-2, Src family kinase or Syk, or both, phosphorylates tyrosine in the hemi-immunoreceptor tyrosine-based activation motif in the cytoplasmic domain of CLEC-2. Tyrosine phosphorylation is recognized by Syk through its two Src homology 2 (SH2) domains, which results in Syk activation. Activated Syk phosphorylates the LAT or SLP-76 adaptor proteins, which induces the activation of effector enzymes, PLC $\gamma 2$, and Btk, which leads to platelet aggregation $[78,80]$.

CLEC-2 expression occurs in platelets, megakaryocytes, neutrophils, monocytes, granulocytes, myeloid, and dendritic cells [81-85]. CLEC-2 null mice reportedly die during the embryonic and neonatal stages with blood-filled lymphatic vessels and edema resulting from defects in blood lymphatic vessel separation [43]. This phenotype is similar to that of podoplanin null mice $[43,86]$. However, platelet-specific and megakaryocyte-specific CLEC-2-deficient mice show no embryonic lethality despite having defects in blood lymphatic vessel separation and mild thrombocytopenia [46, 87]. Thus, CLEC-2 expressed in cells other than platelets may play a crucial role in maintaining life at the embryonic and neonatal stages [87]. Podoplanin-CLEC-2 interaction occurs mainly during development and in pathological situations such as tumors. And importantly for targeting podoplanin-CLEC-2 interaction as a therapy, CLEC-2-deficient platelets remain in the activation pathway stimulated by physiological agonists such as thrombin, ADP, and collagen [86]. However, we need to care about indication that podoplanin-CLEC-2 interaction has a crucial role in the moderation of immune response.

\section{Targeting podoplanin-CLEC-2 interactions for cancer therapy}

As described above, the formation of tumor cell-platelet aggregates is central to the process of hematogenous metastasis. The administration of anti-platelet agents to suppress platelet aggregation can increase bleeding risk significantly in cancer patients with thrombocytopenia due to chemotherapeutic drug toxicity. On the other hand, the interaction between podoplanin on tumor cells and CLEC-2 on platelets is hopeful target for suppressing metastasis of podoplanin-positive tumors, because CLEC-2 null platelets show aggregation induced by physiological agonists such as thrombin, ADP, and collagen [86].

Our laboratory and others have developed a variety of antipodoplanin antibodies to neutralize podoplanin-CLEC-2 interactions and thereby suppress podoplanin-mediated platelet aggregation and hematogenous pulmonary metastasis [75, 88-91]. As human podoplanin uses both PLAG3 and PLAG4 domains for CLEC-2 binding, podoplanin mAbs exhibiting neutralizing ability recognize region in part of PLAG3 or PLAG4 [75]. The monoclonal antibody NZ-1, which recognizes human podoplanin PLAG3, has shown neutralizing activity for podoplanin-CLEC2 interaction, podoplanin-dependent platelet aggregation, and metastasis $[88,92]$. Other established anti-human podoplanin mAbs, P2-0 and MS-1 recognizing the PLAG3 and PG4D2/PG4D2 recognizing PLAG4, suppress platelet aggregation and metastasis by limiting CLEC-2 interaction [75, 90, 91]. Established podoplanin $\mathrm{mAbs}$ indicate suppressive effect against platelet aggregation induced by podoplanin-expressed $\mathrm{CHO}$ [75] and some podoplaninpositive tumor cells $[67,91]$. Moreover, some podoplanin mAbs reportedly show the suppression of in vivo hematogenous metastasis not only using podoplanin-expressed $\mathrm{CHO}$ cells but also using podoplanin-positive human tumor cell lines [19, 51]. These accumulated evidences indicate that podoplanin mAbs could be developed for clinical use. However, the verification using endogenously podoplanin-expressing tumor cells is still restricted. Available cell lines expressing podoplanin from public cell bank are not many, though podoplanin is expressed on a variety of tumors, which is shown by analyses using clinical samples. And, those cell lines are rarely proper for in vivo metastasis model. Actually, when we searched podoplanin-positive lung SCC cell lines which are public available, it turned out that only one cell line PC-10 out of 10 cell lines exhibits podoplanin expression (Fig. 2), even though clinical samples of lung SCCs show more than $60 \%$ podoplanin-positive lung SCC cell lines [93]. This suggests that the characteristics of some tumor cells are changed in the process for establishment of cell lines. Recently, we are trying to establish patient-derived cell lines for podoplanin-positive lung SCC and have succeeded to get several lines. The platelet aggregation induced by established podoplanin-positive lung SCC cells is suppressed by anti-podoplanin mAb PG4D2 strongly than by MS-1 (Fig. 3). Analyses using patient-derived model are also required.

As mentioned in Sect. 3, podoplanin-induced platelet aggregation resulted in the release of platelet factors which could promote tumor progression. In fact, the release of PDGF and TGF- $\beta$ during podoplanin-positive tumor cell-induced platelet aggregation is suppressed by anti-podoplanin mAbs. And the hematogenous metastasis of urinary bladder SCC cell line, UM$\mathrm{UC}-5$, is suppressed by the administration of anti-podoplanin $\mathrm{mAb}$ PG4D and also by that of anti-TGF- $\beta$ mAb 1D11 (Fig. 4). This might indicate that podoplanin-promoted metastasis pathway depends on TGF- $\beta$ mainly at least in UM-UC-5. 


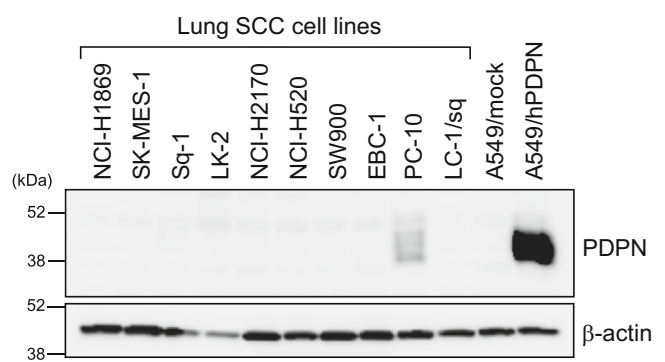

Fig. 2 Search for podoplanin-positive lung SCC cell lines. Public available lung SCC cell lines were analyzed by immunoblotting using anti-podoplanin mAb D2-40 (PDPN) and anti- $\beta$-actin mAb. Nine out of ten lung SCC cell lines are podoplanin negative except PC-10. This frequency is very low compared with the reported result indicated by immunohistochemistry using clinical samples. The discrepancy of existing cell lines from clinical samples is a critical issue for the evaluation of the tool targeting podoplanin

Therefore, searching the downstream pathway of podoplanin leading to metastasis is important for the development of effective strategy for therapy with targeting podoplanin.

Some established anti-podoplanin mAbs, for example, MS-1 and PG4D2, are identified as mouse IgG2a subtype, which exhibits antibody-dependent cellular cytotoxicity/complementdependent cytotoxicity (ADCC/CDC) activities. At least, MS-1
mAb exhibits anti-tumor activity against podoplanin-positive PC10 xenograft tumor in immune-deficient NOD-SCID mouse [91], and the recombinant single-chain antibody variable region fragment of MS-1 also suppresses podoplanin-mediated metasiasis [89]. Therefore, the neutralizing ability of anti-podoplanin $\mathrm{mAb}$ against podoplanin-CLEC-2 interaction may be enough for suppressing metasiasis or growth of podoplanin-positive tumors. Some anti-podoplanin $\mathrm{mAb}$ requires $\mathrm{ADCC} / \mathrm{CDC}$ activities for suppression of tumor growth and metastasis [94, 95]. We cannot ignore the dependency of ADCC/CDC activities of antipodoplanin $\mathrm{mAbs}$ as podoplanin is expressed in several normal tissues and has some physiological roles (see Sect. 3) and need to care the side effect in developing anti-podoplanin $\mathrm{mAb}$ as a therapeutic drug. In that sense, LpMab series, cancer-specific antibodies recognizing cancerous aberrant glycosylated podoplaninan, are potent for therapeutics [96]. Some anti-podoplanin mAbs may be useful as diagnostic tools to identify patients with podoplanin-positive tumor, as higher sensitivity than generally used D2-40, anti-podoplanin mAb [97].

Against many anti-podoplanin mAbs have been established, tools targeting CLEC-2 are only few reported. Targeting CLEC-2 on platelets rather than podoplanin on tumor requires a care for platelet function in hemostasis. A small-molecule compound,
FACS
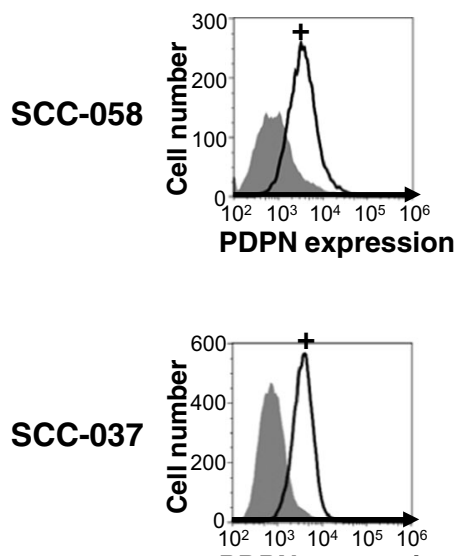

PDPN expression

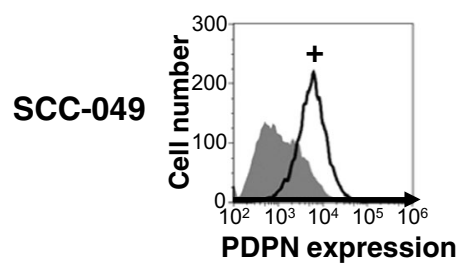

Fig. 3 Patient-derived lung SCC cell-induced platelet aggregation depends on podoplanin. Examples of podoplanin-positive lung SCC cells newly established from clinical samples of patients provided informed consent are shown. Their podoplanin (PDPN) expression was analyzed by flow cytometry (FACS) using anti-podoplanin mAb D2-40. Closed areas indicate negative control without $\mathrm{mAb}$, and open areas indicate D2-40-treated cells; both are treated with anti-mouse IgG-
Platelet aggregation assay
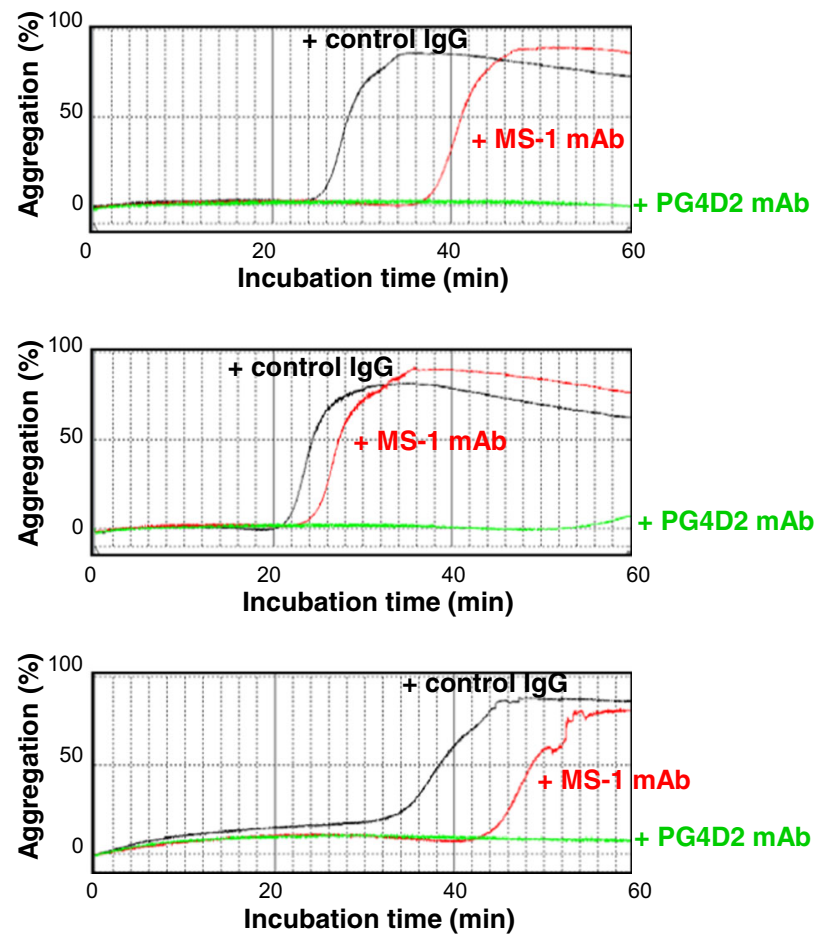

Alexa 488 as a secondary antibody for detection. In the platelet aggregation assays, mouse washed platelets were used. SCC cellinduced platelet aggregation was suppressed by the treatment of cells with anti-podoplanin mAbs (MS-1 and PG4D2) before the addition into assays. Although there are some differences in suppressive effect between MS-1 and PG4D2, and between cells, podoplanin-positive lung SCC cellinduced platelet aggregation depends on podoplanin 


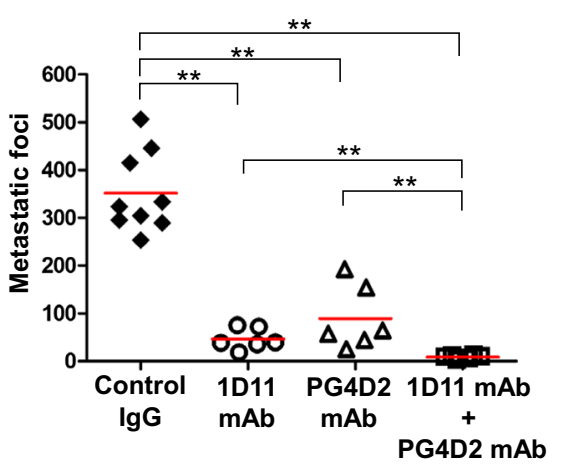

Fig. 4 Hematogenous metastasis of urinary bladder SCC cell UM-UC-5 was significantly suppressed by the administration of anti-podoplanin $\mathrm{mAb}$ PG4D2 and anti-TGF- $\beta$ mAb 1D11. Control IgG or PG4D2 mAb $(30 \mu \mathrm{g} /$ mouse) was intravenously (i.v.) injected into tail vein of CB17SCID mice ( 0,5 weeks) on 1 day before the cell transplantation. Control IgG or $1 \mathrm{D} 11 \mathrm{mAb}(100 \mu \mathrm{g} /$ mouse $)$ was i.v. injected at an hour before the transplantation, and then, UM-UC-5 cells $\left(5 \times 10^{5} /\right.$ mouse $)$ was i.v. injected. After 27 days from the cell transplantation, lungs were excised and the metastatic foci on the surface counted after picric acid staining. Mann-Whitney $U$ tests were performed. $* * P<0.01$. Bars represent mean values. Hematogenous metastasis of UM-UC-5 cells depends on podoplanin and TGF- $\beta$

$2 \mathrm{CP}$, which is a derivative of 4-O-benzoyl-3-methoxy-betanitrostyrene, has been reported as a chemical inhibitor of podoplanin-induced platelet aggregation [98]. 2CP exhibits direct binding activity to CLEC-2 and therapeutic efficacy in combination treatment with cisplatin in a mouse metastasis model without causing defects in physiological platelet function in hemostasis. In another recent report, immunological depletion of CLEC-2 by the treatment of mice with anti-CLEC- $2 \mathrm{mAb}$ 2A2B10 exhibited suppression of hematogenous metastasis and thrombus formation of podoplanin-positive mouse melanoma cell B16F10 without significant bleeding tendency [99].

More analyses using podoplanin-positive tumor cells and tumor/metastasis model containing the patient-derived model are required to provide insights into the development of new therapies targeting the podoplanin-CLEC-2 interaction.

Acknowledgements Authors were supported by the Project for Cancer Research and Therapeutic Evolution (P-CREATE, No. 17cm0106205h0002; to NF) and Medical Research and Development Programs Focused on Technology Transfer, Acceleration Transformative Research for Medical Innovation (ACT-MS, No. $17 \mathrm{im} 0210607 \mathrm{~h} 0002$; to NF) from the Japan Agency for Medical Research and Development (AMED).

We would like to apologize for not citing every related report as references.

\section{Compliance with ethical standards}

Conflict of interest NF received a research grant from API Co., Ltd.; NF holds a patent on anti-podoplanin mAbs.

Open Access This article is distributed under the terms of the Creative Commons Attribution 4.0 International License (http:// creativecommons.org/licenses/by/4.0/), which permits unrestricted use, distribution, and reproduction in any medium, provided you give appropriate credit to the original author(s) and the source, provide a link to the Creative Commons license, and indicate if changes were made.

\section{References}

1. Menter, D. G., Tucker, S. C., Kopetz, S., Sood, A. K., Crissman, J. D., \& Honn, K. V. (2014). Platelets and cancer: a casual or causal relationship: revisited. Cancer and Metastasis Reviews, 33(1), 231269. doi:10.1007/s10555-014-9498-0.

2. Gay, L. J., \& Felding-Habermann, B. (2011). Contribution of platelets to tumour metastasis. Nature Reviews: Cancer, 11(2), 123-134. doi: $10.1038 / \mathrm{nrc} 3004$.

3. Key, N. S., Khorana, A. A., Mackman, N., McCarty, O. J., White, G. C., Francis, C. W., et al. (2016). Thrombosis in cancer: research priorities identified by a National Cancer Institute/National Heart, Lung, and Blood Institute Strategic Working Group. Cancer Research, 76(13), 3671-3675. doi:10.1158/0008-5472.CAN-15-3100.

4. Mehta, P. (1984). Potential role of platelets in the pathogenesis of tumor metastasis. Blood, 63(1), 55-63.

5. Tsuruo, T., Iida, H., Makishima, F., Yamori, T., Kawabata, H., Tsukagoshi, S., et al. (1985). Inhibition of spontaneous and experimental tumor metastasis by the calcium antagonist verapamil. Cancer Chemotherapy and Pharmacology, 14(1), 30-33.

6. Algra, A. M., \& Rothwell, P. M. (2012). Effects of regular aspirin on long-term cancer incidence and metastasis: a systematic comparison of evidence from observational studies versus randomised trials. Lancet Oncology, 13(5), 518-527. doi:10.1016/S14702045(12)70112-2.

7. Rothwell, P. M., Fowkes, F. G., Belch, J. F., Ogawa, H., Warlow, C. P., \& Meade, T. W. (2011). Effect of daily aspirin on long-term risk of death due to cancer: analysis of individual patient data from randomised trials. Lancet, 377(9759), 31-41. doi:10.1016/S01406736(10)62110-1.

8. Tsuruo, T., Yamori, T., Naganuma, K., Tsukagoshi, S., \& Sakurai, Y. (1983). Characterization of metastatic clones derived from a metastatic variant of mouse colon adenocarcinoma 26. Cancer Research, 43(11), 5437-5442.

9. Mahalingam, M., Ugen, K. E., Kao, K. J., \& Klein, P. A. (1988). Functional role of platelets in experimental metastasis studied with cloned murine fibrosarcoma cell variants. Cancer Research, 48(6), 1460-1464.

10. Fidler, I. J. (2003). The pathogenesis of cancer metastasis: the 'seed and soil' hypothesis revisited. Nature Reviews: Cancer, 3(6), 453458. doi:10.1038/nrc1098.

11. Nieswandt, B., Hafner, M., Echtenacher, B., \& Mannel, D. N. (1999). Lysis of tumor cells by natural killer cells in mice is impeded by platelets. Cancer Research, 59(6), 1295-1300.

12. Placke, T., Salih, H. R., \& Kopp, H. G. (2012). GITR ligand provided by thrombopoietic cells inhibits NK cell antitumor activity. Journal of Immunology, 189(1), 154-160. doi:10.4049/jimmunol.1103194.

13. Placke, T., Orgel, M., Schaller, M., Jung, G., Rammensee, H. G., Kopp, H. G., et al. (2012). Platelet-derived MHC class I confers a pseudonormal phenotype to cancer cells that subverts the antitumor reactivity of natural killer immune cells. Cancer Research, 72(2), 440-448. doi:10.1158/0008-5472.CAN-11-1872.

14. Gersuk, G. M., Westermark, B., Mohabeer, A. J., Challita, P. M., Pattamakom, S., \& Pattengale, P. K. (1991). Inhibition of human natural killer cell activity by platelet-derived growth factor (PDGF)III. Membrane binding studies and differential biological effect of recombinant PDGF isoforms. Scandinavian Journal of Immunology, 33(5), 521-532. 
15. Kopp, H. G., Placke, T., \& Salih, H. R. (2009). Platelet-derived transforming growth factor-beta down-regulates NKG2D thereby inhibiting natural killer cell antitumor reactivity. Cancer Research, 69(19), 7775-7783. doi:10.1158/0008-5472.CAN-09-2123.

16. Schumacher, D., Strilic, B., Sivaraj, K. K., Wettschureck, N., \& Offermanns, S. (2013). Platelet-derived nucleotides promote tumorcell transendothelial migration and metastasis via $\mathrm{P} 2 \mathrm{Y} 2$ receptor. Cancer Cell, 24(1), 130-137. doi:10.1016/j.ccr.2013.05.008.

17. Labelle, M., Begum, S., \& Hynes, R. O. (2014). Platelets guide the formation of early metastatic niches. Proceedings of the National Academy of Sciences of the United States of America, 111(30), E3053-E3061. doi:10.1073/pnas.1411082111.

18. Labelle, M., Begum, S., \& Hynes, R. O. (2011). Direct signaling between platelets and cancer cells induces an epithelialmesenchymal-like transition and promotes metastasis. Cancer Cell, 20(5), 576-590. doi:10.1016/j.ccr.2011.09.009.

19. Takemoto, A., Okitaka, M., Takagi, S., Takami, M., Sato, S., Nishio, M., et al. (2017). A critical role of platelet TGF-beta release in podoplanin-mediated tumour invasion and metastasis. Scientific Reports, 7, 42186. doi:10.1038/srep42186.

20. Boucharaba, A., Serre, C. M., Gres, S., Saulnier-Blache, J. S., Bordet, J. C., Guglielmi, J., et al. (2004). Platelet-derived lysophosphatidic acid supports the progression of osteolytic bone metastases in breast cancer. Journal of Clinical Investigation, 114(12), 1714-1725. doi:10.1172/JCI22123.

21. Watanabe, M., Okochi, E., Sugimoto, Y., \& Tsuruo, T. (1988). Identification of a platelet-aggregating factor of murine colon adenocarcinoma 26: Mr 44,000 membrane protein as determined by monoclonal antibodies. Cancer Research, 48(22), 6411-6416.

22. Watanabe, M., Sugimoto, Y., \& Tsuruo, T. (1990). Expression of a Mr 41,000 glycoprotein associated with thrombin-independent platelet aggregation in high metastatic variants of murine B16 melanoma. Cancer Research, 50(20), 6657-6662.

23. Sugimoto, Y., Watanabe, M., Oh-hara, T., Sato, S., Isoe, T., \& Tsuruo, T. (1991). Suppression of experimental lung colonization of a metastatic variant of murine colon adenocarcinoma 26 by a monoclonal antibody 8F11 inhibiting tumor cell-induced platelet aggregation. Cancer Research, 51(3), 921-925.

24. Toyoshima, M., Nakajima, M., Yamori, T., \& Tsuruo, T. (1995). Purification and characterization of the platelet-aggregating sialoglycoprotein gp44 expressed by highly metastatic variant cells of mouse colon adenocarcinoma 26. Cancer Research, 55(4), 767-773.

25. Kato, Y., Fujita, N., Kunita, A., Sato, S., Kaneko, M., Osawa, M., et al. (2003). Molecular identification of Aggrus/T1alpha as a platelet aggregation-inducing factor expressed in colorectal tumors. Journal of Biological Chemistry, 278(51), 51599-51605. doi:10. 1074/jbc.M309935200.

26. Kaneko, M. K., Kato, Y., Kitano, T., \& Osawa, M. (2006). Conservation of a platelet activating domain of Aggrus/ podoplanin as a platelet aggregation-inducing factor. Gene, 378, 52-57. doi:10.1016/j.gene.2006.04.023.

27. Ma, T., Yang, B., Matthay, M. A., \& Verkman, A. S. (1998). Evidence against a role of mouse, rat, and two cloned human t1 alpha isoforms as a water channel or a regulator of aquaporin-type water channels. American Journal of Respiratory Cell and Molecular Biology, 19(1), 143-149. doi:10.1165/ajrcmb.19.1.2953.

28. Zimmer, G., Lottspeich, F., Maisner, A., Klenk, H. D., \& Herrler, G. (1997). Molecular characterization of gp40, a mucin-type glycoprotein from the apical plasma membrane of Madin-Darby canine kidney cells (type I). Biochemical Journal, 326(Pt 1), 99-108.

29. Zimmer, G., Oeffner, F., Von Messling, V., Tschernig, T., Groness, H. J., Klenk, H. D., et al. (1999). Cloning and characterization of gp36, a human mucin-type glycoprotein preferentially expressed in vascular endothelium. Biochemical Journal, 341(Pt 2), 277-284.

30. Nose, K., Saito, H., \& Kuroki, T. (1990). Isolation of a gene sequence induced later by tumor-promoting $12-\mathrm{O}$ - tetradecanoylphorbol-13-acetate in mouse osteoblastic cells (MC3T3-E1) and expressed constitutively in ras-transformed cells. Cell Growth and Differentiation, 1(11), 511-518.

31. Farr, A. G., Berry, M. L., Kim, A., Nelson, A. J., Welch, M. P., \& Aruffo, A. (1992). Characterization and cloning of a novel glycoprotein expressed by stromal cells in T-dependent areas of peripheral lymphoid tissues. Journal of Experimental Medicine, 176(5), $1477-1482$.

32. Schacht, V., Dadras, S. S., Johnson, L. A., Jackson, D. G., Hong, Y. K., \& Detmar, M. (2005). Up-regulation of the lymphatic marker podoplanin, a mucin-type transmembrane glycoprotein, in human squamous cell carcinomas and germ cell tumors. American Journal of Pathology, 166(3), 913-921. doi:10.1016/S0002-9440(10)62311-5.

33. Gandarillas, A., Scholl, F. G., Benito, N., Gamallo, C., \& Quintanilla, M. (1997). Induction of PA2.26, a cell-surface antigen expressed by active fibroblasts, in mouse epidermal keratinocytes during carcinogenesis. Molecular Carcinogenesis, 20(1), 10-18.

34. Breiteneder-Geleff, S., Soleiman, A., Kowalski, H., Horvat, R., Amann, G., Kriehuber, E., et al. (1999). Angiosarcomas express mixed endothelial phenotypes of blood and lymphatic capillaries: podoplanin as a specific marker for lymphatic endothelium. American Journal of Pathology, 154(2), 385-394. doi:10.1016/ S0002-9440(10)65285-6.

35. Rishi, A. K., Joyce-Brady, M., Fisher, J., Dobbs, L. G., Floros, J., VanderSpek, J., et al. (1995). Cloning, characterization, and development expression of a rat lung alveolar type I cell gene in embryonic endodermal and neural derivatives. Developmental Biology, 167(1), 294-306.

36. Breiteneder-Geleff, S., Matsui, K., Soleiman, A., Meraner, P., Poczewski, H., Kalt, R., et al. (1997). Podoplanin, novel 43-kd membrane protein of glomerular epithelial cells, is downregulated in puromycin nephrosis. American Journal of Pathology, 151(4), 1141-1152.

37. Malhotra, D., Fletcher, A. L., Astarita, J., Lukacs-Kornek, V., Tayalia, P., Gonzalez, S. F., et al. (2012). Transcriptional profiling of stroma from inflamed and resting lymph nodes defines immunological hallmarks. Nature Immunology, 13(5), 499-510. doi:10. 1038/ni.2262.

38. Shibahara, J., Kashima, T., Kikuchi, Y., Kunita, A., \& Fukayama, M. (2006). Podoplanin is expressed in subsets of tumors of the central nervous system. Virchows Archiv, 448(4), 493-499. doi: 10.1007/s00428-005-0133-x.

39. Mahtab, E. A., Wijffels, M. C., Van Den Akker, N. M., Hahurij, N. D., Lie-Venema, H., Wisse, L. J., et al. (2008). Cardiac malformations and myocardial abnormalities in podoplanin knockout mouse embryos: correlation with abnormal epicardial development. Developmental Dynamics, 237(3), 847-857. doi:10.1002/dvdy.21463.

40. Ramirez, M. I., Millien, G., Hinds, A., Cao, Y., Seldin, D. C., \& Williams, M. C. (2003). T1alpha, a lung type I cell differentiation gene, is required for normal lung cell proliferation and alveolus formation at birth. Developmental Biology, 256(1), 61-72.

41. Schacht, V., Ramirez, M. I., Hong, Y. K., Hirakawa, S., Feng, D., Harvey, N., et al. (2003). T1alpha/podoplanin deficiency disrupts normal lymphatic vasculature formation and causes lymphedema. EMBO Journal, 22(14), 3546-3556. doi:10.1093/emboj/cdg342.

42. Uhrin, P., Zaujec, J., Breuss, J. M., Olcaydu, D., Chrenek, P., Stockinger, H., et al. (2010). Novel function for blood platelets and podoplanin in developmental separation of blood and lymphatic circulation. Blood, 115(19), 3997-4005. doi:10.1182/blood2009-04-216069.

43. Hess, P. R., Rawnsley, D. R., Jakus, Z., Yang, Y., Sweet, D. T., Fu, J., et al. (2014). Platelets mediate lymphovenous hemostasis to maintain blood-lymphatic separation throughout life. Journal of Clinical Investigation, 124(1), 273-284. doi:10.1172/JCI70422.

44. Herzog, B. H., Fu, J., Wilson, S. J., Hess, P. R., Sen, A., McDaniel, J. M., et al. (2013). Podoplanin maintains high endothelial venule 
integrity by interacting with platelet CLEC-2. Nature, 502(7469), 105-109. doi:10.1038/nature12501.

45. Acton, S. E., Farrugia, A. J., Astarita, J. L., Mourao-Sa, D., Jenkins, R. P., Nye, E., et al. (2014). Dendritic cells control fibroblastic reticular network tension and lymph node expansion. Nature, 514(7523), 498-502. doi:10.1038/nature13814.

46. Tamura, S., Suzuki-Inoue, K., Tsukiji, N., Shirai, T., Sasaki, T., Osada, M., et al. (2016). Podoplanin-positive periarteriolar stromal cells promote megakaryocyte growth and proplatelet formation in mice by CLEC-2. Blood, 127(13), 1701-1710. doi:10.1182/blood2015-08-663708.

47. Hatakeyama, K., Kaneko, M. K., Kato, Y., Ishikawa, T., Nishihira, K., Tsujimoto, Y., et al. (2012). Podoplanin expression in advanced atherosclerotic lesions of human aortas. Thrombosis Research, 129(4), e70-e76. doi:10.1016/j.thromres.2012.01.003.

48. Peters, A., Pitcher, L. A., Sullivan, J. M., Mitsdoerffer, M., Acton, S. E., Franz, B., et al. (2011). Th17 cells induce ectopic lymphoid follicles in central nervous system tissue inflammation. Immunity, 35(6), 986-996. doi:10.1016/j.immuni.2011.10.015.

49. Peterziel, H., Muller, J., Danner, A., Barbus, S., Liu, H. K., Radlwimmer, B., et al. (2012). Expression of podoplanin in human astrocytic brain tumors is controlled by the PI3K-AKT-AP-1 signaling pathway and promoter methylation. Neuro-Oncology, 14(4), 426-439. doi:10.1093/neuonc/nos055.

50. Kunita, A., Kashima, T. G., Ohazama, A., Grigoriadis, A. E., \& Fukayama, M. (2011). Podoplanin is regulated by AP-1 and promotes platelet aggregation and cell migration in osteosarcoma. American Journal of Pathology, 179(2), 1041-1049. doi:10.1016/ j.ajpath.2011.04.027.

51. Takagi, S., Oh-hara, T., Sato, S., Gong, B., Takami, M., \& Fujita, N. (2014). Expression of Aggrus/podoplanin in bladder cancer and its role in pulmonary metastasis. International Journal of Cancer, 134(11), 2605-2614. doi:10.1002/ijc.28602.

52. Kimura, N., \& Kimura, I. (2005). Podoplanin as a marker for mesothelioma. Pathology International, 55(2), 83-86. doi:10.1111/j. 1440-1827.2005.01791.x.

53. Kato, Y., Sasagawa, I., Kaneko, M., Osawa, M., Fujita, N., \& Tsuruo, T. (2004). Aggrus: a diagnostic marker that distinguishes seminoma from embryonal carcinoma in testicular germ cell tumors. Oncogene, 23(52), 8552-8556. doi:10.1038/sj.onc. 1207869.

54. Hisakane, K., Saruwatari, K., Fujii, S., Kirita, K., Umemura, S., Matsumoto, S., et al. (2016). Unique intravascular tumor microenvironment predicting recurrence of lung squamous cell carcinoma. Journal of Cancer Research and Clinical Oncology, 142(3), 593600. doi:10.1007/s00432-015-2068-1.

55. Mishima, K., Kato, Y., Kaneko, M. K., Nishikawa, R., Hirose, T., \& Matsutani, M. (2006). Increased expression of podoplanin in malignant astrocytic tumors as a novel molecular marker of malignant progression. Acta Neuropathologica, 111(5), 483-488. doi:10. 1007/s00401-006-0063-y.

56. Mishima, K., Kato, Y., Kaneko, M. K., Nakazawa, Y., Kunita, A., Fujita, N., et al. (2006). Podoplanin expression in primary central nervous system germ cell tumors: a useful histological marker for the diagnosis of germinoma. Acta Neuropathologica, 111(6), 563568. doi:10.1007/s00401-006-0033-4.

57. Yuan, P., Temam, S., El-Naggar, A., Zhou, X., Liu, D. D., Lee, J. J., et al. (2006). Overexpression of podoplanin in oral cancer and its association with poor clinical outcome. Cancer, 107(3), 563-569. doi:10.1002/cncr.22061.

58. Kawase, A., Ishii, G., Nagai, K., Ito, T., Nagano, T., Murata, Y., et al. (2008). Podoplanin expression by cancer associated fibroblasts predicts poor prognosis of lung adenocarcinoma. International Journal of Cancer, 123(5), 1053-1059. doi:10.1002/ijc.23611.

59. Hoshino, A., Ishii, G., Ito, T., Aoyagi, K., Ohtaki, Y., Nagai, K., et al. (2011). Podoplanin-positive fibroblasts enhance lung adenocarcinoma tumor formation: podoplanin in fibroblast functions for tumor progression. Cancer Research, 71(14), 4769-4779. doi:10. 1158/0008-5472.CAN-10-3228.

60. Pula, B., Jethon, A., Piotrowska, A., Gomulkiewicz, A., Owczarek, T., Calik, J., et al. (2011). Podoplanin expression by cancerassociated fibroblasts predicts poor outcome in invasive ductal breast carcinoma. Histopathology, 59(6), 1249-1260. doi:10.1111/ j.1365-2559.2011.04060.x.

61. Yurugi, Y., Wakahara, M., Matsuoka, Y., Sakabe, T., Kubouchi, Y., Haruki, T., et al. (2017). Podoplanin expression in cancerassociated fibroblasts predicts poor prognosis in patients with squamous cell carcinoma of the lung. Anticancer Research, 37(1), 207213. doi:10.21873/anticanres.11308.

62. Yoshida, T., Ishii, G., Goto, K., Neri, S., Hashimoto, H., Yoh, K., et al. (2015). Podoplanin-positive cancer-associated fibroblasts in the tumor microenvironment induce primary resistance to EGFR-TKIs in lung adenocarcinoma with EGFR mutation. Clinical Cancer Research, 21(3), 642-651. doi:10.1158/1078-0432.CCR-14-0846.

63. Hatzioannou, A., Nayar, S., Gaitanis, A., Barone, F., Anagnostopoulos, C., \& Verginis, P. (2016). Intratumoral accumulation of podoplanin-expressing lymph node stromal cells promote tumor growth through elimination of CD4+ tumor-infiltrating lymphocytes. Oncoimmunology, 5(9), e1216289. doi:10.1080/ $2162402 X .2016 .1216289$.

64. Suzuki, H., Onimaru, M., Koga, T., Takeshita, M., Yano, T., Maehara, Y., et al. (2011). High podoplanin expression in cancer cells predicts lower incidence of nodal metastasis in patients with lung squamous cell carcinoma. Pathology, Research and Practice, 207(2), 111-115. doi:10.1016/j.prp.2010.11.006.

65. Ito, T., Ishii, G., Nagai, K., Nagano, T., Kojika, M., Murata, Y., et al. (2009). Low podoplanin expression of tumor cells predicts poor prognosis in pathological stage IB squamous cell carcinoma of the lung, tissue microarray analysis of 136 patients using 24 antibodies. Lung Cancer, 63(3), 418-424. doi:10.1016/j.lungcan.2008.06.008.

66. Ikoma, Y., Kijima, H., Masuda, R., Tanaka, M., Inokuchi, S., \& Iwazaki, M. (2015). Podoplanin expression is correlated with the prognosis of lung squamous cell carcinoma. Biomedical Research (Tokyo, Japan), 36(6), 393-402. doi:10.2220/biomedres.36.393.

67. Kunita, A., Kashima, T. G., Morishita, Y., Fukayama, M., Kato, Y., Tsuruo, T., et al. (2007). The platelet aggregation-inducing factor aggrus/podoplanin promotes pulmonary metastasis. American Journal of Pathology, 170(4), 1337-1347. doi:10.2353/ajpath. 2007.060790.

68. Takagi, S., Takemoto, A., Takami, M., Oh-Hara, T., \& Fujita, N. (2014). Platelets promote osteosarcoma cell growth through activation of the platelet-derived growth factor receptor-Akt signaling axis. Cancer Science, 105(8), 983-988. doi:10.1111/cas.12464.

69. Miyata, K., Takemoto, A., Okumura, S., Nishio, M., \& Fujita, N. (2017) Podoplanin enhances lung cancer cell growth in vivo by inducing platelet aggregation. Scientific Reports, 7, 4059. doi:10. 1038/s41598-017-04324-1.

70. Wicki, A., Lehembre, F., Wick, N., Hantusch, B., Kerjaschki, D., \& Christofori, G. (2006). Tumor invasion in the absence of epithelialmesenchymal transition: podoplanin-mediated remodeling of the actin cytoskeleton. Cancer Cell, 9(4), 261-272. doi:10.1016/j.ccr. 2006.03.010.

71. Kaneko, M., Kato, Y., Kunita, A., Fujita, N., Tsuruo, T., \& Osawa, M. (2004). Functional sialylated O-glycan to platelet aggregation on Aggrus (T1alpha/Podoplanin) molecules expressed in Chinese hamster ovary cells. Journal of Biological Chemistry, 279(37), 38838-38843. doi:10.1074/jbc.M407210200.

72. Kaneko, M. K., Kato, Y., Kameyama, A., Ito, H., Kuno, A., Hirabayashi, J., et al. (2007). Functional glycosylation of human podoplanin: glycan structure of platelet aggregation-inducing factor. FEBS Letters, 581(2), 331-336. doi:10.1016/j.febslet.2006.12.044.

73. Amano, K., Chiba, Y., Kasahara, Y., Kato, Y., Kaneko, M. K., Kuno, A., et al. (2008). Engineering of mucin-type human 
glycoproteins in yeast cells. Proceedings of the National Academy of Sciences of the United States of America, 105(9), 3232-3237. doi:10.1073/pnas.0710412105.

74. Nagae, M., Morita-Matsumoto, K., Kato, M., Kaneko, M. K., Kato, Y., \& Yamaguchi, Y. (2014). A platform of C-type lectin-like receptor CLEC-2 for binding O-glycosylated podoplanin and nonglycosylated rhodocytin. Structure, 22(12), 1711-1721. doi: 10.1016/j.str.2014.09.009.

75. Sekiguchi, T., Takemoto, A., Takagi, S., Takatori, K., Sato, S., Takami, M., et al. (2016). Targeting a novel domain in podoplanin for inhibiting platelet-mediated tumor metastasis. Oncotarget, 7(4), 3934-3946. doi:10.18632/oncotarget.6598.

76. Huang, T. F., Liu, C. Z., \& Yang, S. H. (1995). Aggretin, a novel platelet-aggregation inducer from snake (Calloselasma rhodostoma) venom, activates phospholipase $\mathrm{C}$ by acting as a glycoprotein Ia/IIa agonist. Biochemical Journal, 309(Pt 3), 10211027.

77. Suzuki-Inoue, K., Fuller, G. L., Garcia, A., Eble, J. A., Pohlmann, S., Inoue, O., et al. (2006). A novel Syk-dependent mechanism of platelet activation by the C-type lectin receptor CLEC-2. Blood, 107(2), 542-549. doi:10.1182/blood-2005-05-1994.

78. Suzuki-Inoue, K., Kato, Y., Inoue, O., Kaneko, M. K., Mishima, K., Yatomi, Y., et al. (2007). Involvement of the snake toxin receptor CLEC-2, in podoplanin-mediated platelet activation, by cancer cells. Journal of Biological Chemistry, 282(36), 25993-26001. doi:10.1074/jbc.M702327200.

79. Christou, C. M., Pearce, A. C., Watson, A. A., Mistry, A. R., Pollitt, A. Y., Fenton-May, A. E., et al. (2008). Renal cells activate the platelet receptor CLEC-2 through podoplanin. Biochemical Journal, 411(1), 133-140. doi:10.1042/BJ20071216.

80. Fuller, G. L., Williams, J. A., Tomlinson, M. G., Eble, J. A., Hanna, S. L., Pohlmann, S., et al. (2007). The C-type lectin receptors CLEC-2 and Dectin-1, but not DC-SIGN, signal via a novel YXXL-dependent signaling cascade. Journal of Biological Chemistry, 282(17), 12397-12409. doi:10.1074/jbc.M609558200.

81. Kerrigan, A. M., Dennehy, K. M., Mourao-Sa, D., Faro-Trindade, I., Willment, J. A., Taylor, P. R., et al. (2009). CLEC-2 is a phagocytic activation receptor expressed on murine peripheral blood neutrophils. Journal of Immunology, 182(7), 4150-4157. doi:10.4049/ jimmunol.0802808.

82. Colonna, M., Samaridis, J., \& Angman, L. (2000). Molecular characterization of two novel C-type lectin-like receptors, one of which is selectively expressed in human dendritic cells. European Journal of Immunology, 30(2), 697-704. doi:10.1002/1521-4141(200002) 30:2<697::AID-IMMU697>3.0.CO;2-M.

83. Chang, C. H., Chung, C. H., Hsu, C. C., Huang, T. Y., \& Huang, T. F. (2010). A novel mechanism of cytokine release in phagocytes induced by aggretin, a snake venom C-type lectin protein, through CLEC-2 ligation. Journal of Thrombosis and Haemostasis, 8(11), 2563-2570. doi:10.1111/j.1538-7836.2010.04045.x.

84. Mourao-Sa, D., Robinson, M. J., Zelenay, S., Sancho, D., Chakravarty, P., Larsen, R., et al. (2011). CLEC-2 signaling via Syk in myeloid cells can regulate inflammatory responses. European Journal of Immunology, 41(10), 3040-3053. doi:10. 1002/eji.201141641.

85. Acton, S. E., Astarita, J. L., Malhotra, D., Lukacs-Kornek, V., Franz, B., Hess, P. R., et al. (2012). Podoplanin-rich stromal networks induce dendritic cell motility via activation of the C-type lectin receptor CLEC-2. Immunity, 37(2), 276-289. doi:10.1016/j. immuni.2012.05.022.

86. Suzuki-Inoue, K., Inoue, O., Ding, G., Nishimura, S., Hokamura, K., Eto, K., et al. (2010). Essential in vivo roles of the C-type lectin receptor CLEC-2: embryonic/neonatal lethality of CLEC-2deficient mice by blood/lymphatic misconnections and impaired thrombus formation of CLEC-2-deficient platelets. Journal of
Biological Chemistry, 285(32), 24494-24507. doi:10.1074/jbc. M110.130575.

87. Osada, M., Inoue, O., Ding, G., Shirai, T., Ichise, H., Hirayama, K., et al. (2012). Platelet activation receptor CLEC-2 regulates blood/ lymphatic vessel separation by inhibiting proliferation, migration, and tube formation of lymphatic endothelial cells. Journal of Biological Chemistry, 287(26), 22241-22252. doi:10.1074/jbc. M111.329987.

88. Kato, Y., Kaneko, M. K., Kuno, A., Uchiyama, N., Amano, K., Chiba, Y., et al. (2006). Inhibition of tumor cell-induced platelet aggregation using a novel anti-podoplanin antibody reacting with its platelet-aggregation-stimulating domain. Biochemical and Biophysical Research Communications, 349(4), 1301-1307. doi: 10.1016/j.bbrc.2006.08.171.

89. Miyata, K., Takagi, S., Sato, S., Morioka, H., Shiba, K., Minamisawa, T., et al. (2014). Suppression of Aggrus/ podoplanin-induced platelet aggregation and pulmonary metastasis by a single-chain antibody variable region fragment. Cancer Medicine, 3(6), 1595-1604. doi:10.1002/cam4.320.

90. Nakazawa, Y., Takagi, S., Sato, S., Oh-hara, T., Koike, S., Takami, M., et al. (2011). Prevention of hematogenous metastasis by neutralizing mice and its chimeric anti-Aggrus/podoplanin antibodies. Cancer Science, 102(11), 2051-2057. doi:10.1111/j.1349-7006. 2011.02058.x.

91. Takagi, S., Sato, S., Oh-hara, T., Takami, M., Koike, S., Mishima, Y., et al. (2013). Platelets promote tumor growth and metastasis via direct interaction between Aggrus/podoplanin and CLEC-2. PloS One, 8(8), e73609. doi:10.1371/journal.pone.0073609.

92. Kato, Y., Kaneko, M. K., Kunita, A., Ito, H., Kameyama, A., Ogasawara, S., et al. (2008). Molecular analysis of the pathophysiological binding of the platelet aggregation-inducing factor podoplanin to the C-type lectin-like receptor CLEC-2. Cancer Science, 99(1), 54-61. doi:10.1111/j.1349-7006.2007.00634.x.

93. Kato, Y., Kaneko, M., Sata, M., Fujita, N., Tsuruo, T., \& Osawa, M. (2005). Enhanced expression of Aggrus (T1alpha/podoplanin), a platelet-aggregation-inducing factor in lung squamous cell carcinoma. Tumour Biology, 26(4), 195-200. doi:10.1159/000086952.

94. Kato, Y., Kunita, A., Abe, S., Ogasawara, S., Fujii, Y., Oki, H., et al. (2015). The chimeric antibody chLpMab-7 targeting human podoplanin suppresses pulmonary metastasis via $\mathrm{ADCC}$ and $\mathrm{CDC}$ rather than via its neutralizing activity. Oncotarget, 6(34), 3600336018. doi:10.18632/oncotarget.5339.

95. Kato, Y., Kunita, A., Fukayama, M., Abe, S., Nishioka, Y., Uchida, H., et al. (2017). Antiglycopeptide mouse monoclonal antibody LpMab-21 exerts antitumor activity against human podoplanin through antibody-dependent cellular cytotoxicity and complementdependent cytotoxicity. Monoclon Antib Immunodiagn Immunother, 36(1), 20-24. doi:10.1089/mab.2016.0045.

96. Kato, Y., \& Kaneko, M. K. (2014). A cancer-specific monoclonal antibody recognizes the aberrantly glycosylated podoplanin. Scientific Reports, 4, 5924. doi:10.1038/srep05924.

97. Kaneko, M. K., Oki, H., Ogasawara, S., Takagi, M., \& Kato, Y. (2015). Anti-podoplanin monoclonal antibody LpMab-7 detects metastatic lesions of osteosarcoma. Monoclon Antib Immunodiagn Immunother, 34(3), 154-161. doi:10.1089/mab. 2014.0091.

98. Chang, Y. W., Hsieh, P. W., Chang, Y. T., Lu, M. H., Huang, T. F., Chong, K. Y., et al. (2015). Identification of a novel platelet antagonist that binds to CLEC-2 and suppresses podoplanin-induced platelet aggregation and cancer metastasis. Oncotarget, 6(40), 42733-42748. doi:10.18632/oncotarget.5811.

99. Shirai, T., Inoue, O., Tamura, S., Tsukiji, N., Sasaki, T., Endo, H., et al. (2017). C-type lectin-like receptor 2 promotes hematogenous tumor metastasis and prothrombotic state in tumor-bearing mice. Journal of Thrombosis and Haemostasis, 15(3), 513-525. doi:10. 1111/jth.13604. 